\title{
A genome-wide RNAi screen identifies novel targets of neratinib sensitivity leading to neratinib and paclitaxel combination drug treatments $\dagger$
}

\author{
Attila A. Seyhan, $\ddagger^{* a}$ Usha Varadarajan, ${ }^{a}$ Sung Choe,${ }^{b}$ Yan Liu, ${ }^{a}$ John McGraw, ${ }^{a}$ \\ Matthew Woods, ${ }^{a}$ Stuart Murray, $\rfloor^{b}$ Amy Eckert, ${ }^{a}$ Wei Liu $\ddagger^{*}{ }^{b}$ and \\ Terence E. Ryan ${ }^{c}$
}

Received 22nd November 2010, Accepted 3rd March 2011

DOI: $10.1039 / \mathrm{c0mb00294a}$

ErbB2 is frequently activated in tumors, and influences a wide array of cellular functions, including proliferation, apoptosis, cell motility and adhesion. HKI-272 (neratinib) is a small molecule pan-kinase inhibitor of the ErbB family of receptor tyrosine kinases, and shows strong antiproliferative activity in ErbB2-overexpressing breast cancer cells. We undertook a genome-wide pooled lentiviral RNAi screen to identify synthetic lethal or enhancer (synthetic modulator screen) genes that interact with neratinib in a human breast cancer cell line (SKBR-3). These genes upon knockdown would modulate cell viability in the presence of subeffective concentrations of neratinib. We discovered a diverse set of genes whose depletion selectively impaired or enhanced the viability of SKBR-3 cells in the presence of neratinib. We observed diverse pathways including EGFR, hypoxia, cAMP, and protein ubiquitination that, when co-treated with RNAi and neratinib, resulted in arrest of cell proliferation. Examining the changes of these genes and their protein products also led to a rationale for clinically relevant drug combination treatments. Treatment of cells with either paclitaxel or cytarabine in combination with neratinib resulted in a strong antiproliferative effect. The identification of novel mediators of cellular response to neratinib and the development of potential drug combination treatments have expanded our understanding of neratinib's mode-of-action for the development of more effective therapeutic regimens. Notably, our findings support a paclitaxel and neratinib phase III clinical trial in breast cancer patients.

\section{Introduction}

Activation of intracellular mitogenic signal transduction pathways driven by the ErbB family of receptor tyrosine kinases has been implicated in a variety of cancers. Amongst these, the tumorigenic roles of the Epidermal Growth Factor Receptor (EGFR) and ErbB2 (HER-2/Neu) have been most extensively studied. Activation of ErbB2 occurs primarily by overexpression in breast, ovarian, lung, prostate, gastric and oral cancers, leading to spontaneous homodimerization and

${ }^{a}$ Systems Biology, Global Biotherapeutics, Pfizer Inc.,

87 Cambridgepark Drive, Cambridge, MA 02140.

E-mail:attila.seyhan@pfizer.com,attila_seyhan@yahoo.com,

wei.liu@agios.com

${ }^{b}$ Agios Pharmaceuticals, Cambridge, MA 02139, USA

${ }^{c}$ iBio, Inc., 9 Innovation Way, Newark, DE 19711, USA

$\dagger$ Electronic supplementary information (ESI) available. See DOI: $10.1039 / \mathrm{c} 0 \mathrm{mb} 00294 \mathrm{a}$

$\ddagger$ Current address: Pfizer Inc., Translational Immunology, Inflammation and Immunology, 200 Cambridgepark Drive, Cambridge, MA 02140, USA. activation of downstream signaling in a ligand-independent manner. The role of ErbB2 has been most thoroughly documented in breast cancer, where it is overexpressed in $25-30 \%$ of cases, and is correlated with a poor prognosis. ${ }^{1-3}$

Breast cancers can be classified into those that express the estrogen (ER) and progesterone (PR) receptors, those with ErbB2 amplification, and those without expression of ER, PR, or amplification of ErbB2 (referred to as triple-negative or basal-like breast cancer). ${ }^{4}$ Therefore, it is a heterogeneous group of diseases. Approximately $60-70 \%$ of breast cancers express estrogen receptors (ER) and/or progesterone receptors (PR), and approximately $20-30 \%$ of breast cancers have amplified ErbB2 thereby leading to high levels of the ErbB2 protein. ${ }^{4-6}$ In approximately $15-20 \%$ of patients with breast cancer, the tumors do not express ER or PR and do not have amplification of ErbB2. ${ }^{5}$ These are classified as triple-negative breast cancer. Patients carrying these tumors have a poor prognosis. ${ }^{5}$ Expression profiling of primary breast cancers and breast cancer cell lines has determined that the majority of these triple-negative tumors show similar expression profiles 
with basal epithelial cells of the breast duct $^{7-10}$ and hence are also referred to as basal-like tumors. The current treatment regimen for these tumors is therapeutic antibodies and small molecule kinase inhibitors targeting EGFR for the treatment of colon and lung cancer, respectively. ${ }^{3}$

Herceptin (trastuzumab), a monoclonal antibody, has shown success for ErbB2-directed breast cancer therapy and benefits patients in both the adjuvant and metastatic settings. Herceptin was initially approved for the treatment of women with metastatic breast cancer overexpressing ErbB2. ${ }^{11}$ Studies conducted in metastatic breast cancer patients demonstrated that herceptin alone had a finite and measurable response rate. ${ }^{12,13}$ Although in the adjuvant setting for early-stage ErbB2-positive breast cancer patients, herceptin therapy after chemotherapy offers significant long-term therapeutic benefit, ${ }^{14}$ clinically not all patients benefit from ErbB2 antagonist therapies and a subset of patients show disease progression after an initial response. The efficacy of herceptin depends on the ErbB2 status of the tumor and the patient's prior treatment, but even when patients are selected on the basis of ErbB2 gene amplification, the single-agent response rate ranges from 12 to $30 \% .{ }^{15}$ There is thus an urgent need for the development of more potent drugs targeting the ErbB family members.

HKI-272 (neratinib) ${ }^{3,16}$ is a potent, low molecular weight, orally administered irreversible pan-ErbB (ErbB1, 2, and 4) receptor tyrosine kinase inhibitor that covalently binds to the cytoplasmic domain of ErbB proteins and inhibits their autophosphorylation and subsequent phosphorylation of downstream substrates. ${ }^{3,16-18}$ Neratinib suppresses the proliferation of cells that overexpress ErbB2 ( $\left.\mathrm{IC}_{50} 2-3 \mathrm{nM}\right)$ and EGFR $\left(\mathrm{IC}_{50} 81 \mathrm{nM}\right),{ }^{3,16}$ leading to (a) the inhibition of downstream signal transduction event, (b) arrest of the cell cycle at the G1-S-phase transition, and (c) decreased cellular proliferation. Neratinib has demonstrated antitumor efficacy in phase 1 and 2 clinical studies in patients with advanced ErbB2-positive breast cancer, including those with or without prior herceptin exposure. ${ }^{19,20}$ Neratinib interferes with EGFR and ErbB2 functions by blocking receptor phosphorylation, most likely by binding the cysteine residue in the ATP-binding pocket of the receptor, leading to reduction of autophosphorylation of the receptor. ${ }^{16}$ In preclinical models, neratinib inhibits ErbB receptor complex downstream signaling via the phosphatidylinositol-3-kinase (PI3K) and MAPK pathways. ${ }^{21}$ Activation of the PI3K signaling pathway may mediate resistance to ErbB2 targeted or endocrine therapy in breast cancer because of crosstalk among estrogen receptor, ErbB, and PI3K pathways. $^{21}$ A greater understanding of the mechanism of action of neratinib and potential resistance mechanisms to it in cancer cells would be extremely valuable to develop neoadjuvant regimens. Identification of genes that modify neratinib efficacy could also lead to combination therapies as well as the identification of biomarkers for patient stratification.

RNA interference (RNAi) is a physiological sequencespecific gene silencing process triggered by double-stranded RNA (dsRNA) that can operate both transcriptionally and posttranscriptionally. ${ }^{22-24}$ Recently, RNAi has become a powerful technology in reverse genetics and potentially as therapeutics. For instance, the recent development of RNAi libraries that systematically target every gene in the genome has made it possible to conduct genome scale RNAi screens that probe phenotypes associated with the loss-of-function of many genes simultaneously. ${ }^{25,26}$ By silencing gene expression and thereby protein function, RNAi mimics the pharmacological inhibition of target protein with added benefit that it can also target the non-druggable genome and is therefore a powerful tool to discover and validate novel targets.

Synthetic lethality, first described in yeast genetics, ${ }^{27}$ occurs when alteration of a gene results in change of the cellular phenotype (e.g., arrest of cell proliferation or perturbation of cell viability) only in the presence of another nonlethal genetic alteration. Recently this approach has been applied to mammalian cells ${ }^{28,29}$ and to RNAi screens. ${ }^{30}$ RNAi screens in combination with active compounds were used for the identification of sensitizing targets and novel genetic interdependencies in cancer. $^{26,31-33}$ Synthetic lethal (negative selection) screens have been used to identify RNAi reagents that promote drug sensitivity ${ }^{34,35}$ or cell lethality. ${ }^{36}$ For instance, RNAi screens for genes that show differential requirement between cell lines, and identification of dependency of oncogenic KRAS and STK33 suppression in human cancer cells, have been reported. ${ }^{37,38}$ In addition, synthetic lethal screens in combination with RNAi libraries have been used to identify mechanisms of resistance and sensitivity to specific chemicals including genes whose inhibition sensitized cancer cells to chemotherapeutic agents. ${ }^{33,39,40}$

Here we conducted a genome-wide RNAi and neratinibdependent synthetic lethal screen to identify novel components of ErbB2 signaling and other signaling pathways that specifically reduce cell proliferation or viability in the presence of otherwise subeffective concentrations of neratinib in ErbB2overexpressing human breast cancer cells. Toward this end, we used a pooled lentiviral RNAi approach in which shRNAs that were depleted over time were identified through the competitive hybridization of barcodes derived from the shRNA population to a microarray. ${ }^{34,36,41,42}$ The "depleted shRNAs" corresponding to those genes that conferred selective disadvantage in the presence of the subeffective concentrations of neratinib and hence were only present in the control population were identified. The identification of novel mediators of cellular response to subeffective concentrations of neratinib could lead to the development of potential combination therapies and significantly expand our understanding of neratinib's mechanism of action. Our work presented here demonstrates the utility of whole genome RNAi functional genetic screens for identifying genotype-specific drug sensitizing or resistant genes in human breast cancer cells.

\section{Materials and methods}

\section{Cell culture}

ErbB2-overexpressing SKBR-3 and BT-474 breast cancer cells were grown in Dulbecco's modified Eagle medium (DMEM) supplemented with $10 \%$ fetal bovine serum (FBS), $100 \mathrm{U} \mathrm{mL}^{-1}$ penicillin, and $100 \mu \mathrm{g} \mathrm{mL}^{-1}$ streptomycin. Cells were grown at $37{ }^{\circ} \mathrm{C}$ in $5 \% \mathrm{CO}_{2}$ in $90 \%$ humidity. 


\section{Cell proliferation and viability assays to determine inhibitory concentrations (IC) for neratinib}

Cells were seeded in sextuplet in 96-well plates with neratinib at 24 concentration points, and the medium was retained throughout the experiment or replaced every three days. On days two and nine post-drug treatment, cell proliferation and viability were measured daily using WST (Roche) and CellTiter-Glo reagent (CTG, Promega) per manufacturer's instruction on a Wallac Victor 2 (Perkin Elmer) or Envision Plate Reader (Perkin Elmer), respectively. Cell proliferation and viability were calculated by dividing the average of the RLU values for the drug treated wells by the average of the $\mathrm{RLU}$ values for vehicle treated wells. The $\mathrm{IC}_{50}$ values were determined using a GraphPad Prism (GraphPad Software, San Diego, California, USA) and values were shown as calculated $\mathrm{IC}_{50} \pm 95 \%$ confidence level.

\section{Genome-wide lentiviral-shRNA transduction of SKBR-3 cells and barcode screen}

For the screen, a human genome-wide-pooled lentiviral shRNAmir library (Decode lentiviral-shRNAmir library, Open Biosystems) was used. The library consists of $\sim 74000$ unique mir-30-based shRNAs targeting approximately 18000 human genes distributed into seven sub-pools, each sub-pool consisting of $\sim 10000$ individual shRNAs. SKBR-3 breast cancer cells were transduced with individual seven pools of lentiviral shRNAmir constructs at a multiplicity of infection (MOI) of $\sim 0.3$ to achieve $\sim 1$ integrant per cell and at a redundancy of $\sim 375$ per manufacturers recommendation and ref. 34 and 36 .

Each transduction was performed in three replicates. The transduced cells were subsequently selected with puromycin $\left(2 \mu \mathrm{g} \mathrm{mL}^{-1}\right.$ ) for 14 days $^{43}$ to establish stably integrated cells. Prior to this, we established a puromycin kill curve on SKBR-3 cells and $2 \mu \mathrm{g} \mathrm{mL}{ }^{-1}$ was determined to be the effective concentration for establishing stable cells. The stably selected cells were subsequently divided into two populations, one for control $\left(0.5 \%\right.$ DMSO containing media plus $2 \mu \mathrm{g} \mathrm{mL} \mathrm{m}^{-1}$ puromycin), and the other for exposure to subeffective concentrations of neratinib $\left(\mathrm{IC}_{30} ; 0.393 \mathrm{nM}\right)$ as the selective pressure for five days to induce the synthetic lethal phenotype.

\section{Recovery of chemosensitizing and chemoresistance genes from the RNAi screen}

Following the five-day selection, genomic DNAs were extracted from control and neratinib treated cells using Qiagen's blood and a cell culture DNA Kit (Qiagen, cat \# 13323) from three independent control and treatment replicates $\left(5 \times 10^{6}\right.$ cells per treatment). The isolated genomic DNAs from each of the seven pools were pooled, quantified spectrophotometrically. $3.3 \mu \mathrm{g}$ of genomic DNA from each treatment was used as a template for amplification of the 60 nucleotide barcodes unique to each shRNAmir by PCR using the forward (5'-CAA GGG GCT ACT TTA GGA GCA ATT ATC TTG) and reverse (5'-GGT TGA TTG TTC CAG ACG CGT) primers following manufacturers recommendation (Open Biosystems). PCR products (190 bp) for each replicate were pooled, precipitated, and gel-purified on a $1.2 \%$ agarose gel and correct PCR bands were extracted from gel slices using the Wizard SV Gel and PCR clean up system (Promega, catalog \# A9281) and quantified. PCR products were labeled with cyanine-3 (Cy3) or cyanine-5 (Cy5) fluorescent groups using ULS (Kreatech) and purified over a KreaPure (Kreatech) spin column, following manufacturer's recommendation (Open Biosystems).

\section{Barcode microarray hybridizations}

Decode shRNA barcode microarrays (two sub-arrays of 58498 probes each, Agilent) were used to identify shRNAs that were depleted or enriched in the population. Cy3 and Cy5 labeled PCR products were competitively hybridized to each Decode shRNA barcode microarray with dye-swap following manufacturers recommendation (Open Biosystems). Microarrays were hybridized for 18 hours at $42{ }^{\circ} \mathrm{C}$, washed as per the manufacturer's wash protocol, and scanned using an Agilent microarray scanner. Quantification of the resulting fluorescent images was performed with Imagene 5.6 (BioDiscovery), local background was subtracted, and the data were normalized and $\log 2$ transformed. By comparing the processed signals at each spot (Cy5/Cy3), barcodes (hence shRNAs) that are depleted ("drop outs") or enriched after the selection were determined.

\section{Statistical analyses}

To identify spots with significant differential representation in the control (DMSO) versus neratinib-treated samples, we conducted the statistical calculations using the Bioconductor R package "limma" (http://www.bioconductor.org/packages/ bioc/html/limma.html), choosing a model that included a dye factor and treated technical replicates differently from biological replicates (see pp. 38-39 of the limma User Guide http:// www.bioconductor.org/packages/bioc/vignettes/limma/inst/doc/ usersguide.pdf). To account for multiple hypotheses testing, false discovery rates (FDR) were calculated from the $p$-values using the Benjamini and Yekutieli step-up FDR controlling procedure from Bioconductor's R package "multtest" (http:// bioconductor.org/packages/bioc/html/multtest.html).

\section{Combination drug treatment of SKBR-3 cells}

To measure the effect of drug:drug interaction on cell growth and viability, we performed a factorial dose matrix combinatorial drug treatment with 11 dose points for neratinib and 6 dose points for either paclitaxel, cytarabine, colchicine, or vinblastine in triplicate. After determining single agent activities for all the drugs, we designed dose-matrix experiments with concentration samples centered on each drug's effective inhibitory concentration at $50 \%$ effect $\left(\mathrm{IC}_{50}\right) .{ }^{44}$ Cell cultures $(10000$ cells per well of a 96 well plate) were treated with each compound individually at fixed multiples (0.3-218 $\mathrm{nM}$ for each compound and 0.1-1968 nM for neratinib). Experiments were performed in triplicate. Cells treated with drugs were incubated for five days in an incubator at $37{ }^{\circ} \mathrm{C}$. Combination index (CI) analysis $^{44,45}$ was used to determine the synergy of a combination of each compound with neratinib for five days. Experiments that yielded an average CI value of less than 1 were repeated at least six times to allow for determination of confidence intervals for the CI values obtained. ${ }^{46}$ 
For the calculation of the combination drug treated cells, each well was assigned a viability ratio calculated as mean viability in paclitaxel or paclitaxel + neratinib divided by mean viability in the absence of drug ( mean $\left._{\text {paclitaxel }} / \mathrm{mean}_{\text {vehicle }}\right)$. Analysis of synergism between compounds in inducing growth arrest and cytotoxic effects on cells was done by the median effect method of Chou and Talalay ${ }^{47}$ using the commercially available software (Calcusyn, Biosoft) ${ }^{47}$ and the combination index $(\mathrm{CI})$ values were calculated. ${ }^{47}$

\section{Results}

The objective of this study was to develop a platform technology to rapidly perform genome-wide RNAi "dropout" screens to systematically identify chemosensitizer genes to subeffective concentrations of neratinib. Such a study could also lead to the identification of genes potentially required for cancer cell proliferation and survival, and to the nomination of neoadjuvant or combinatorial drugs treatments. We used our screening platform to interrogate human SKBR-3 ErbB2 over-expressing human breast cancer cells.

\section{Dose response of ErbB2 positive cells to neratinib}

To obtain multiple inhibitory concentrations (IC) values of neratinib, dose response experiments were performed in SKBR-3 and BT-474 ErbB2 positive breast cancer cell lines. The treatment was performed in two formats, either the single dosing or multi-dosing, where dosing was performed every three days by replacing growth media with fresh neratinib. The plates were assayed every day up to 14 days. Our data suggest that there was no significant difference between single and multi-dosing of cells: therefore, single dosing treatment was used in later experiments for higher throughput. Plotting IC values against the time where samples were collected and assayed revealed that after five days, there is a tendency of convergence of neratinib effect on cell proliferation as well as viability irrespective of the concentration of the drug (Fig. 1). This led us to identify day five as the assay window for the treatment of cells with neratinib at subeffective concentration $\left(\mathrm{IC}_{30}, 0.39 \mathrm{nM}\right) . \mathrm{IC}_{30}$ was selected as the subeffective concentrations of the drug for the screen because it provides a window that is considered robust for the approach but still shows no effect on proliferation and viability.

\section{Genome-wide RNAi synthetic lethality screens for mediators of neratinib sensitivity in breast cancer cells}

We used a pooled library of $\sim 74000$ distinct mir-30 based shRNAs expressed from the RNA pol-II promoter from a lentiviral backbone targeting $\sim 18000$ human genes. We determined the functional titers of packaged viruses to be approximately one log lower than that for HEK293 cells determined by the manufacturer. Cells were infected with an average representation of 375 independent integrations per shRNA by a multiplicity of infection of (MOI) 0.3 (Fig. 2). The cells were puromycin-selected $\left(2 \mu \mathrm{g} \mathrm{mL}^{-1}\right)$ and propagated for a total of 14 days, and used for the synthetic lethal screen. After 14 days of puromycin selection for the stable integrants, initial reference genomic DNA samples were collected for barcode amplification before treatment of cells with subeffective concentrations $\left(\mathrm{IC}_{30}, 0.39 \mathrm{nM}\right)$ of neratinib and second
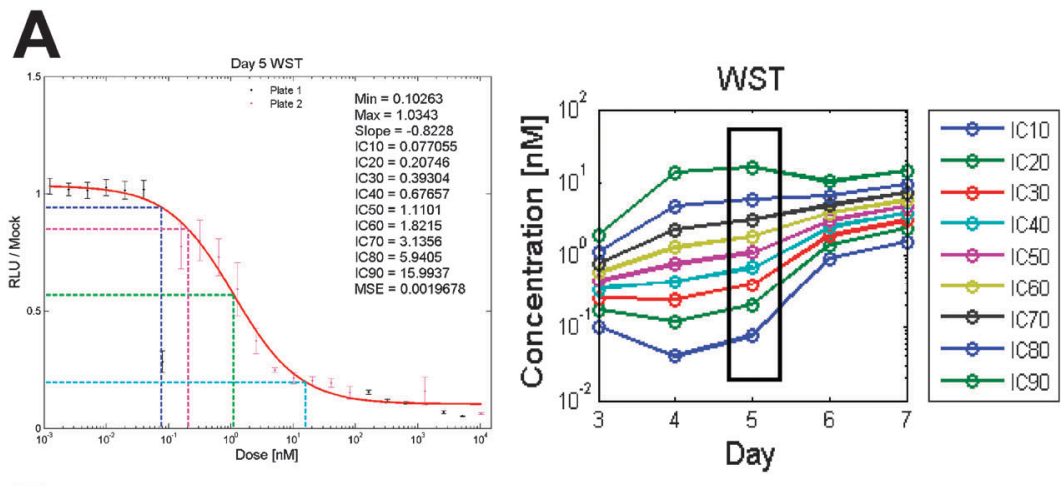

\begin{tabular}{|l|c|c|c|c|c|}
\hline & Day 3 & Day 4 & Day 5 & Day 6 & Day 7 \\
\hline IC10 & 0.102 & 0.040 & 0.077 & 0.902 & 1.552 \\
\hline IC20 & 0.176 & 0.120 & 0.207 & 1.410 & 2.352 \\
\hline IC30 & 0.251 & 0.243 & 0.393 & 1.880 & 3.037 \\
\hline IC40 & 0.335 & 0.432 & 0.676 & 2.428 & 3.798 \\
\hline IC50 & 0.442 & 0.747 & 1.110 & 3.032 & 4.704 \\
\hline IC60 & 0.576 & 1.281 & 1.821 & 3.798 & 5.753 \\
\hline IC70 & 0.768 & 2.278 & 3.135 & 4.904 & 7.195 \\
\hline IC80 & 1.128 & 4.750 & 5.940 & 6.538 & 9.592 \\
\hline IC90 & 1.880 & 13.632 & 15.993 & 10.225 & 14.531 \\
\hline
\end{tabular}
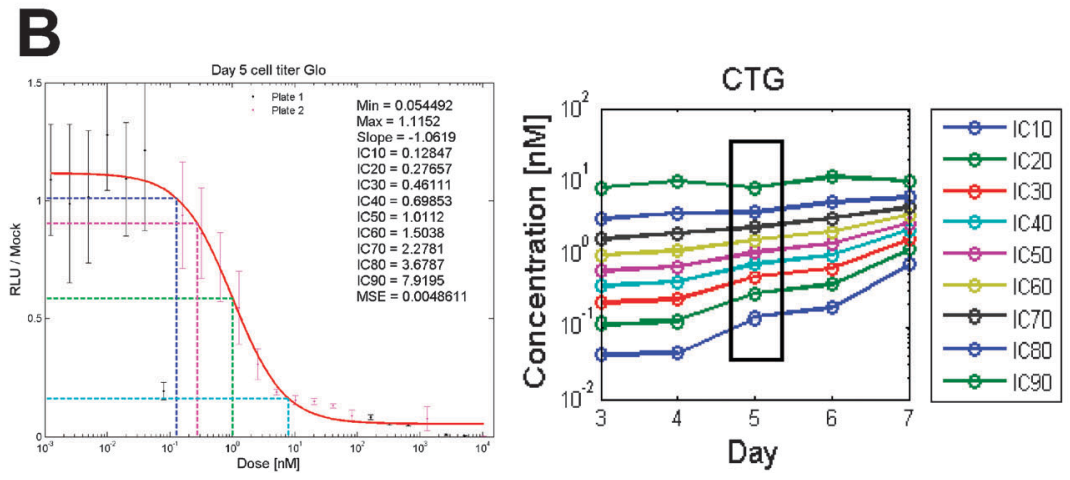

\begin{tabular}{|l|c|c|c|c|c|}
\hline & Day 3 & Day 4 & Day 5 & Day 6 & Day 7 \\
\hline IC10 & 0.040 & 0.043 & 0.128 & 0.171 & 0.698 \\
\hline IC20 & 0.106 & 0.116 & 0.276 & $\mathbf{0 . 3 6 8}$ & 1.128 \\
\hline IC30 & 0.200 & 0.228 & 0.461 & 0.614 & 1.552 \\
\hline IC40 & 0.345 & 0.393 & 0.698 & 0.931 & 2.069 \\
\hline IC50 & 0.563 & 0.650 & 1.011 & 1.370 & 2.611 \\
\hline IC60 & 0.931 & 1.058 & 1.503 & 2.004 & 3.342 \\
\hline IC70 & 1.552 & 1.880 & 2.278 & 3.037 & 4.315 \\
\hline IC80 & 2.941 & 3.563 & 3.678 & 5.063 & 5.940 \\
\hline IC90 & 7.919 & 9.904 & 7.919 & 11.254 & 9.904 \\
\hline
\end{tabular}

Fig. 1 Neratinib dose response effect on cell proliferation. A 24-point dose-response was performed in SKBR-cells in a 96-well plate format and cell proliferation was measured using cell proliferation assay kits (WST, Roche, or CTG, Promega) on individual replicates of plates every day for seven days. The data were fit using sigmoidal curve fit and multiple IC values were determined from the curve fittings. 


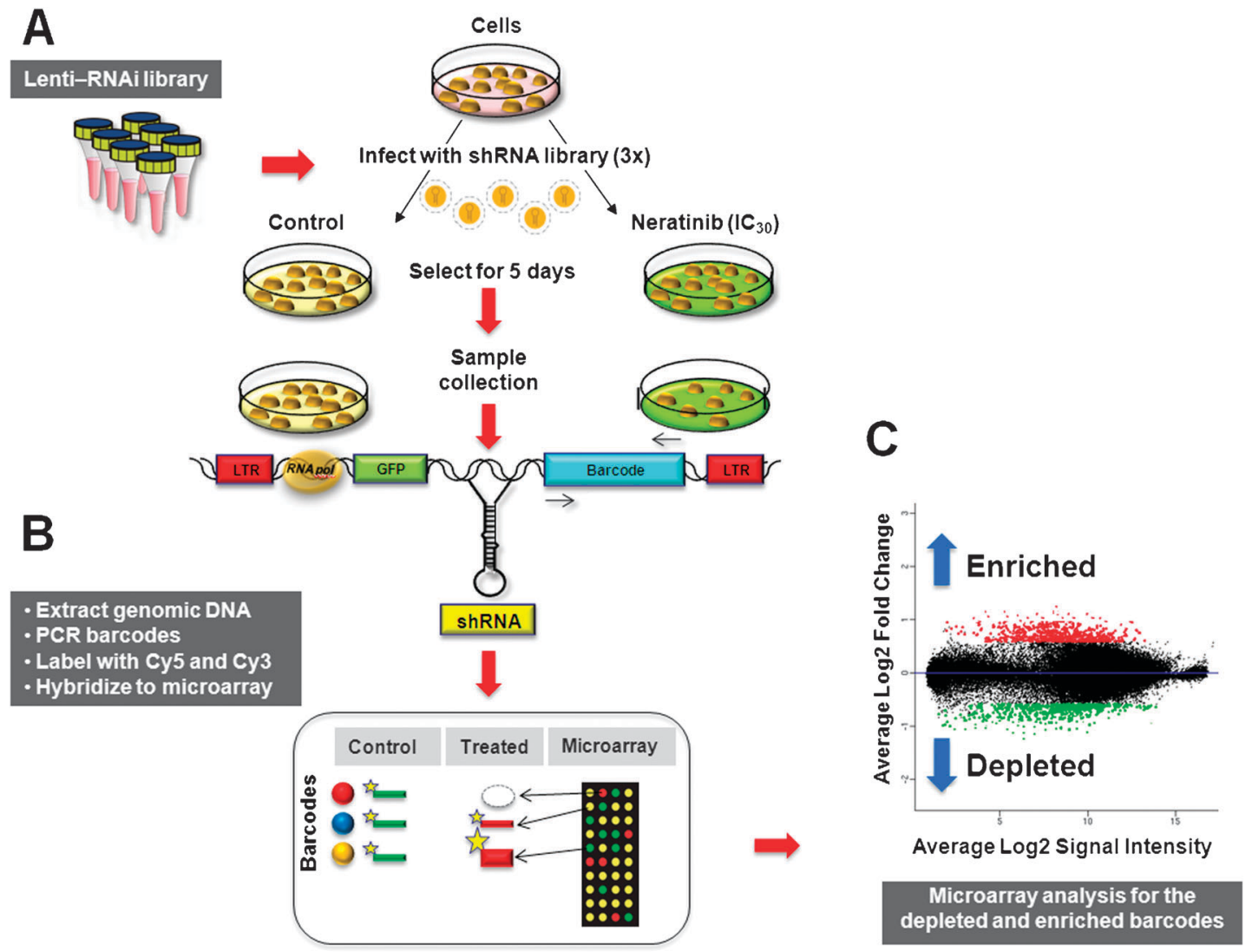

Fig. 2 Synthetic modulator selection screen: identification of drug sensitive genes. Flowchart of the functional human genome-wide lentiviral shRNA-mediated synthetic modulator screen measuring the effect of subeffective concentrations of neratinib upon gene knockdown. (A) For the screen, ErbB2 overexpressing SKBR-3 breast cancer cells was infected with the human genome-wide lentiviral-shRNAmir library at a MOI of $\sim 0.3$ to achieve $\sim 1$ integrant per cell and each individual shRNA occupied $\sim 375$ cells. The infected cells were subsequently selected with puromycin over an $\sim 14$ day culture period and divided into two populations. One is treated (or selected) with a drug or a selective pressure, whereas the other population serves as a reference for the hybridization. The pool of drug selected cell library was treated with neratinib at a sublethal concentration $\left(0.39 \mathrm{nM}, \mathrm{IC}_{30}\right)$ and time points were collected on day zero or day five after drug treatment whereas the other population served as a control. (B). After five days, genomic DNA was isolated and the unique 60-mer random 'molecular barcodes' were recovered by PCR from each population. PCR products were gel purified, labeled with Cy5 and Cy3 dyes, and hybridized to multiplex Decode shRNA barcode microarrays (Agilent) in competition with a common reference to identify the depleted shRNA sequences. The 60-mer barcodes were used to identify the detected knockdown gene in a large population of cells. Since each shRNA is tagged with a unique barcode sequence the frequency (representation) of each shRNA in a mixed population can be measured by hybridizing barcodes to an oligonucleotide microarray. If the population is subjected to a selective pressure such as neratinib drug as in this study, the representation of individual barcodes is expected to change as a consequence which can be measured by comparing hybridization signals for the control population with those of the population subjected to selective pressure. Consequently, the relative signal of shRNAs that sensitize to the cells to selective pressure will decrease, whereas the relative signal of those that increase resistance to the selection pressure will increase. (C) The graph depicts the $\log 2$ of the average intensity versus the log 2 of the average fold change. shRNA vectors that are enriched by the selection method are represented by spots with an increased ratio. Adapted from ref. 31. This graph exemplifies the data obtained from the screen where relative abundance of barcodes recovered from the screen that are enriched by the selection method are represented by spots with an increased ratio. The relative signal of shRNAs that sensitize to the cells to selective pressure will decrease, whereas the relative signal of those that increase resistance to the selection pressure will increase.

genomic DNA samples were collected five days after drug treatment. Furthermore, cells were exposed to drug continuously for five days to allow multiple cell cycles to occur, ${ }^{39}$ allowing effects of synthetic lethality effect (neratinib + RNAi).

\section{Hit identification and data quality assessment}

Sixty nucleotide barcodes were PCR-recovered from genomic DNA, labeled with Cy5 and Cy3 dyes, respectively, and hybridized to a barcode microarray (Fig. 2) per manufacturer's recommendation. The $\mathrm{Cy} 3 / \mathrm{Cy} 5$ signal ratio of each probe reports the change in relative abundance of a particular
shRNA between the beginning and the end of the experiment. Correlations between initial samples across the triplicates and between the initial and end samples within each replica were high (FDR $\leq 0.01$ with an absolute fold change of $>1.5$ ), indicating that the triplicates were highly reproducible and representation was well maintained throughout the experiment. In addition, the consistency between duplicated chips was significantly high $\left(p<1 \times 10^{-5}\right.$ for both depleted and enriched barcodes), and the correlation coefficient did not change significantly when compared between dye-swapped chips (not shown). 


\section{Identification of genes and pathways whose inhibition by RNAi leads to the synthetic lethal effect}

We looked for genes that when absent via RNAi, confer either better survival ("resisters") or early lethality ("sensitizers") in the presence of subeffective concentrations of neratinib. We analyzed data sets using a custom statistical package based on the Linear Models for Microarray data (Limma) method ${ }^{48}$ from Bioconductor for two-color cDNA microarray analysis. ${ }^{34}$

We calculated FDR values using Bioconductor's multitest package and used the Benjamini and Yekutieli step-up FDR controlling procedure. We set the FDR cut-off to $\leq 0.01$ and absolute fold change to $\geq 2$ against the background (lentiviral transduced cell population treated with $0.5 \%$ DMSO) (Fig. 3A and B). We identified 38 unique gene symbols that were depleted (Table 1) and 59 that were enriched (Table 2) in $\mathrm{IC}_{30}$ neratinib treated cells. These genes, when inhibited by RNAi, enhanced death or proliferation of cancer cells in the presence of subeffective concentration of neratinib, respectively, and represented components of core cellular modules essential for the ErbB2 overexpressing SKBR-3 cell line.

Although $\leq 0.01 \%$ FDR is a very rigorous cut-off and could produce false negative of genes that may have functional roles in these cells, ${ }^{41}$ this cut-off has returned many candidates with overlapping functional relationships, including macromolecular complexes, receptor-ligand pairs, and products of related compensatory signaling pathway gene expression products. Most striking was the presence of a large group of testesexpressed genes involved mostly in spermatogenesis. One target encoding membrane protein (transmembrane basic domain; gene symbol, LOC401506 (new LOC648245) was isolated. BEAN (brain expressed, associated with Nedd4) expression was significantly down (2.5 fold change) in the screen, indicating that BEAN knockdown sensitizes SKBR-3 cells to neratinib. It was also shown that BEAN knockdown sensitizes the H1155 lung cancer cell line to paclitaxel. ${ }^{41}$

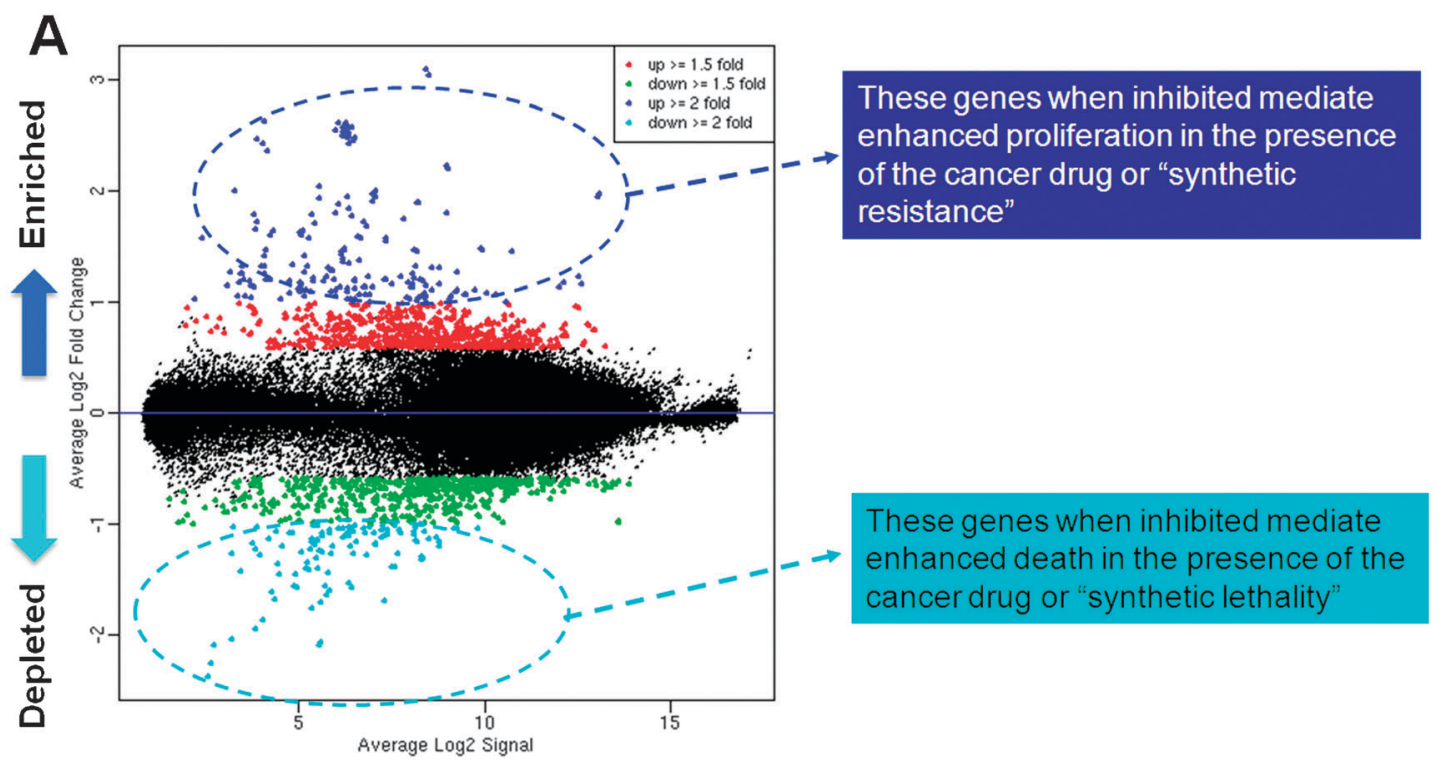

B

Gene list Absolute FC >2

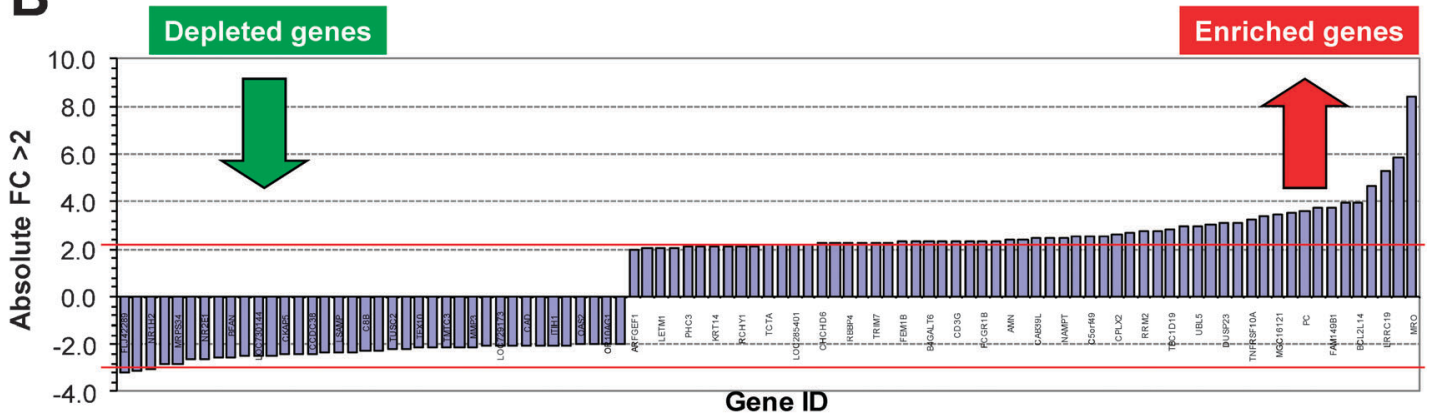

Fig. 3 Neratinib-dependent RNAi screen identified chemosensitizer and chemoresistance genes. (A) The graph depicts the results of the analysis of the relative abundance of barcodes recovered from the screen. The data are the average of three independent barcode samples extracted from three independent drug selected cell populations and three independent hybridization experiments performed in duplicate with reversed color by dye swapping. shRNA expression vectors that are enriched by the selection method are represented by spots with an increased ratio. List of genes targeted by the shRNA vectors were significantly enriched $(P<0.01)$ in subeffective concentrations $\left(\mathrm{IC}_{30}\right)$ neratinib-treated cells. (B) Using cut-offs FDR $\leq 0.01$ and absolute fold change $\geq 2$, we identified 59 unique genes whose knockdown conferred proliferative enrichment of cells (enriched) and 38 unique genes whose knockdown conferred cells chemosensitive (depleted) in combination with neratinib at subeffective concentrations. 


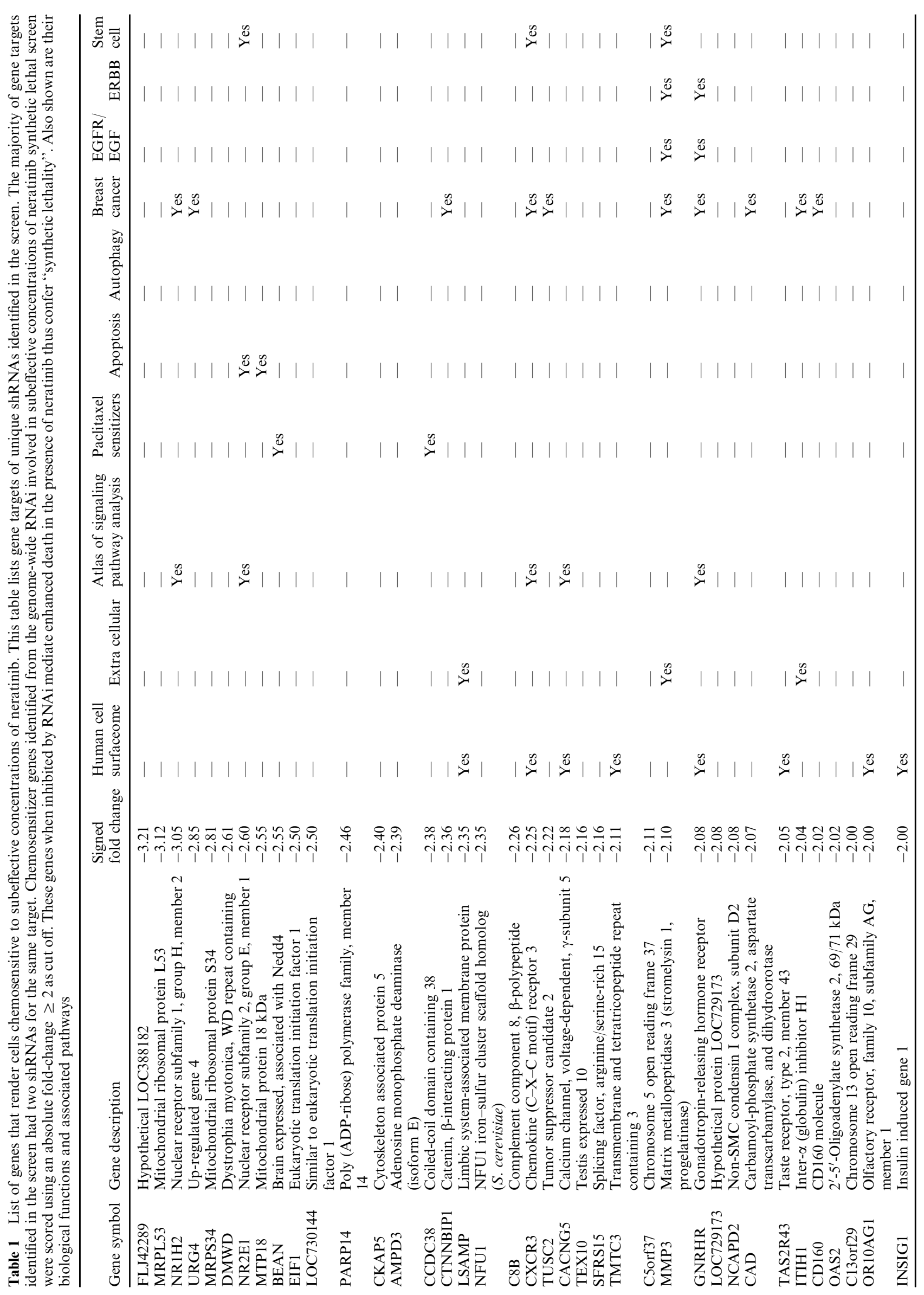




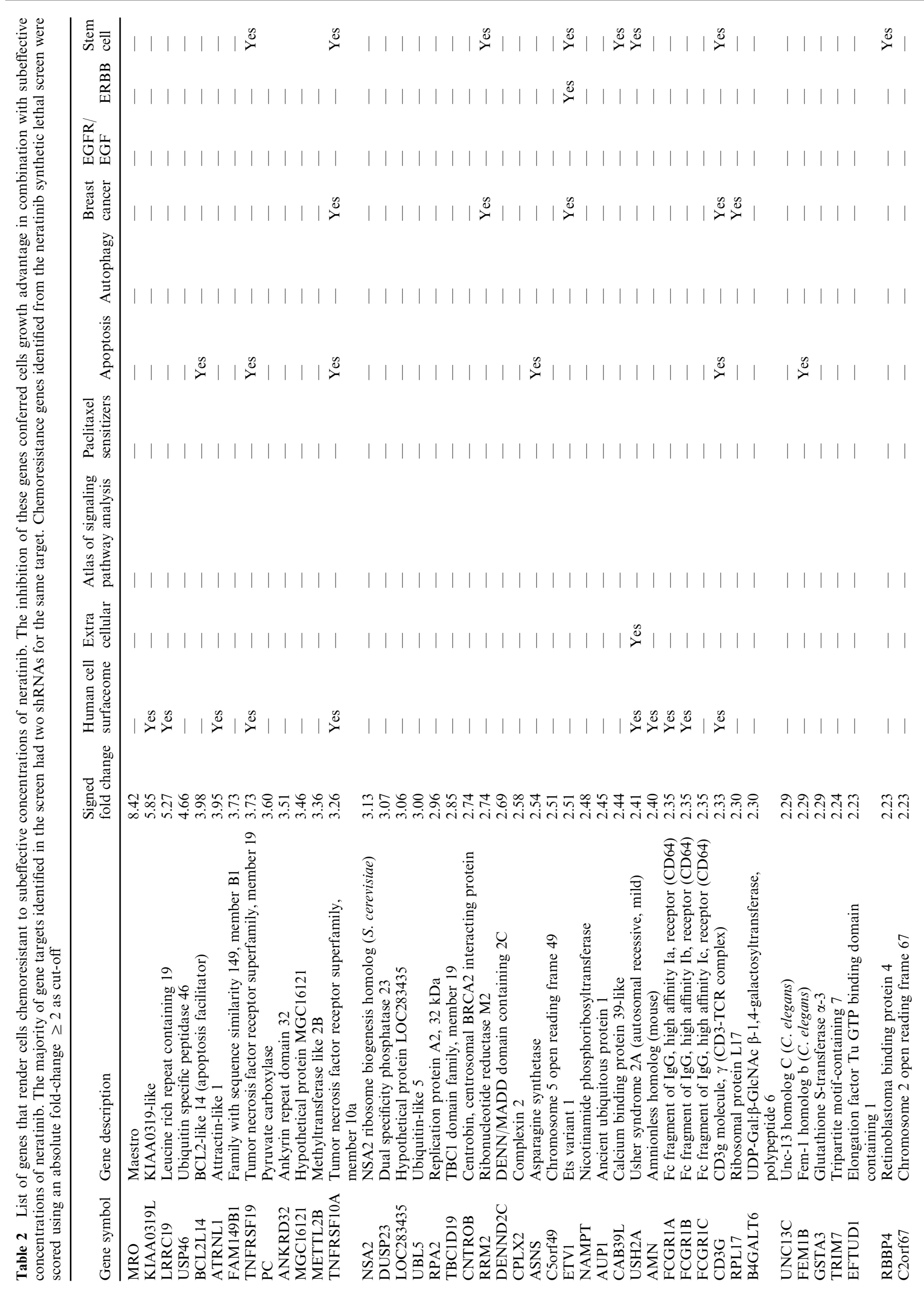




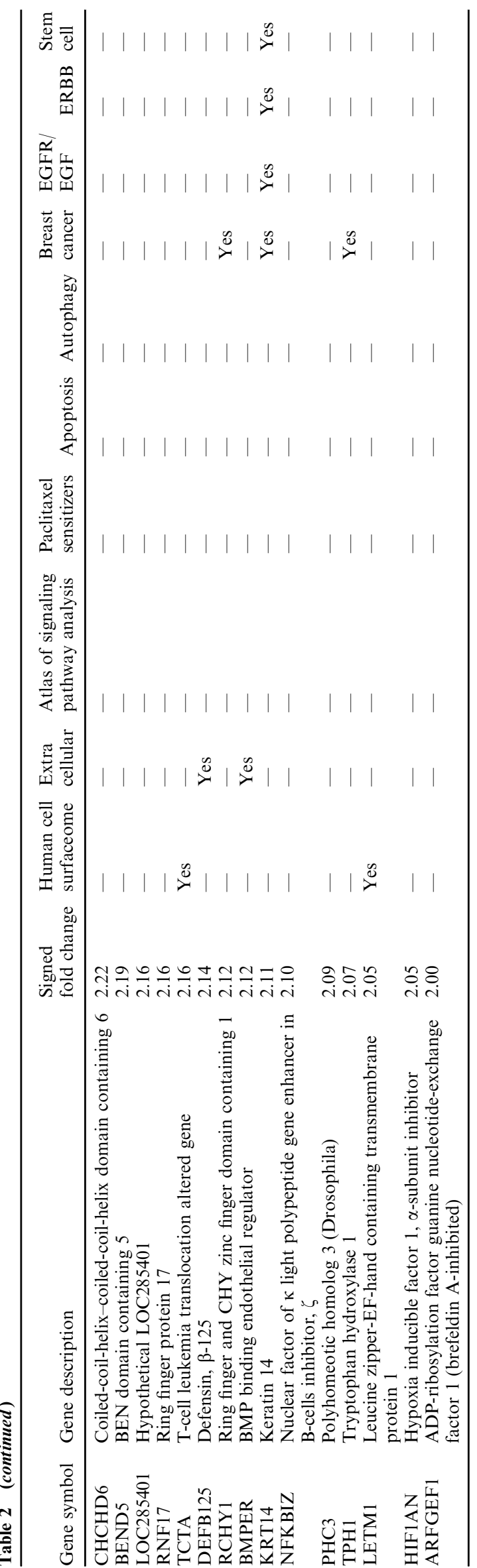

BEAN was identified by interaction with NEDD4 an E3 ubiquitin ligase known to target an epithelial $\mathrm{Na}^{+}$channel and was recently shown to target PTEN. We have also identified three additional genes from our screen previously reported in paclitaxel sensitizer RNAi screen (CCDC38, RNF151, SIPA1L2 with signed fold changes, $-2.55,-2.38$, +1.53 , and +1.52 , respectively). ${ }^{41}$ Since a number of genes identified from this stringent cut-off did not permit for pathway prediction, we relaxed the fold change threshold to 1.5. When the fold change cutoff was loosened to $1.5,397$ gene symbols were enriched and 384 depleted. Pathway analysis of these genes was conducted using Pathway Studio (Ariadne Genomics) and Ingenuity Pathway Analysis (Ingenuity).

Interestingly, many of the barcodes did not have gene assignments as provided by the vendor. We found that some of these barcodes matched EST sequences that were not assigned to Entrez Genes, while others matched intergenic genomic sequences. We have aligned the sense sequences with all RNA sequences for improved annotations where possible.

\section{Identification of key components in signaling pathways}

Relaxing the fold change cut-off to 1.5 returned many additional genes which allowed us to map them to several signal transduction cascades, including hypoxia, cAMP, EFGR, Hedgehog patched/SMO to Myc, alternate EGFR/ Neurophilin/VEGFR, G-protein coupled receptors, insulin receptor (direct and indirect), $\mathrm{Ca}^{2+} / \mathrm{CAM}$ Kinase, and apoptosis (Fig. S1, ESI $\dagger$ ). Most connections are by genes "synthetic lethal" with neratinib and they all converge at the same down-stream "effectors" as in the canonical EGFR pathway (Fig. S1, ESI $\dagger$ ).

We postulate based on these results that therapeutic strategies aimed at suppressing the key components in hypoxia (e.g., NQOi and CREB), cAMP, protein ubiquitination (e.g., PP2B, CaM, AKAP), or others in EGFR (Fig. S2, ESI $\dagger$ ) or ER (Fig. S3, ESI $\dagger$ ) signaling pathways could provide alternative targets. Additionally, 158 genes from the screen can be directly linked to breast cancer by text-mining the PubMed abstracts using Pathway Studio. We have also identified 66 genes from our screen that overlapped with stem cell specific genes, and 44 of them overlapped with genes linked to breast cancer. At least two shRNAs against the same gene scored in the screen, which suggests that their effects are unlikely due to off-target hits. Furthermore, the screen also identified a number of membrane surface proteins and ligands that seem to be involved in local (such as epiregulin) or distal synthetic effects with the drug by converging downstream at the same EGFR pathway "effectors". The discovery of both the "local" and "distal" synthetic effects opens more possibilities for choosing the suitable drug combinations with neratinib for a combinatorial regimen, as well as suitable biomarkers for neratinib resistance.

\section{Identification of compounds that may act synergistically with neratinib}

We performed a comprehensive text mining and pathway mapping of the hit gene products for the identification of possible drug combinations with neratinib. They were analyzed extensively by integrating text-mined directional relationships and 
pathways from the literature, protein-protein interactions from high-throughput screens, and FDA's drug information. More specifically, we examined the output of the Connectivity Map ("cMAP") 49 from the Broad Institute and looked for the compounds that can produce the similar gene expression responses as in our screen. In addition, we used the Pathway Studio tool to look for compounds that can generate the similar gene expression changes as in our screen. Since both cMAP and Pathway Studio tool are standard tools and not an automated process, the final list was created by manual selection based on the commonality of the results from the two tools. By combining the results from these two approaches and via manual curation and selection, we came down to the final list of compounds.
We were mostly interested in identifying drugs that may be immediately used together with neratinib for a combinatorial drug therapy regimen. Therefore we investigated the roles of all pharmaceutical compounds in regulating these genes by text-mining and pathway building studies. From these efforts, we identified cytarabine (genes and respective fold change $((-)$ : down regulated) or (+): up regulated)): MYOG $(-1.8)$, KDR (-1.8), FLI1 (-1.5), GLUL $(+1.6)$, and CDC25A (+1.6) and paclitaxel (KDR (-1.8), NR1I2 $(+1.7)$, ANGPT1 $(+1.8)$, and TNFRSF10A $(+3.3))$ as the top candidates, followed by doxycycline and doxorubicin, then COX-1/COX-2 inhibitors such as indomethacin and the discontinued drug sulindac, and less significantly the
A

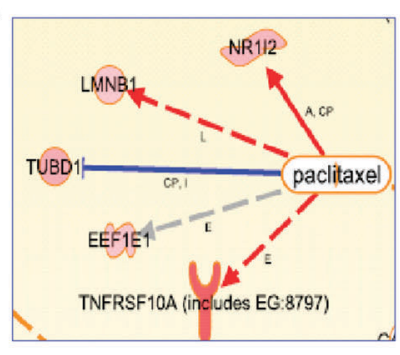

B

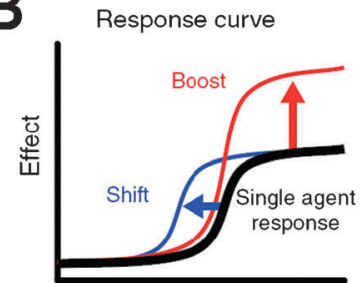

[Drug]

\begin{tabular}{|c|c|c|}
\hline $\begin{array}{l}\text { Gene } \\
\text { symbol }\end{array}$ & Gene description & $\begin{array}{l}\text { Signed fold } \\
\text { change }\end{array}$ \\
\hline BEAN & $\begin{array}{l}\text { Brain expressed, associated } \\
\text { with Nedd } 4\end{array}$ & -2.55 \\
\hline CCD38 & $\begin{array}{l}\text { Coiled-coil domain containing } \\
38\end{array}$ & -2.38 \\
\hline RNF151 & Ring finger protein 151 & +1.53 \\
\hline SIPA1L2 & $\begin{array}{l}\text { Signal-induced proliferation- } \\
\text { associated } 1 \text { like } 2\end{array}$ & +1.52 \\
\hline
\end{tabular}

Dosing ratios

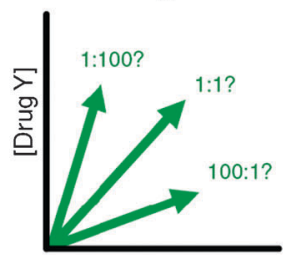

[Drug X]

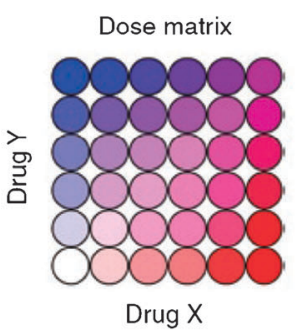

Drug X
C

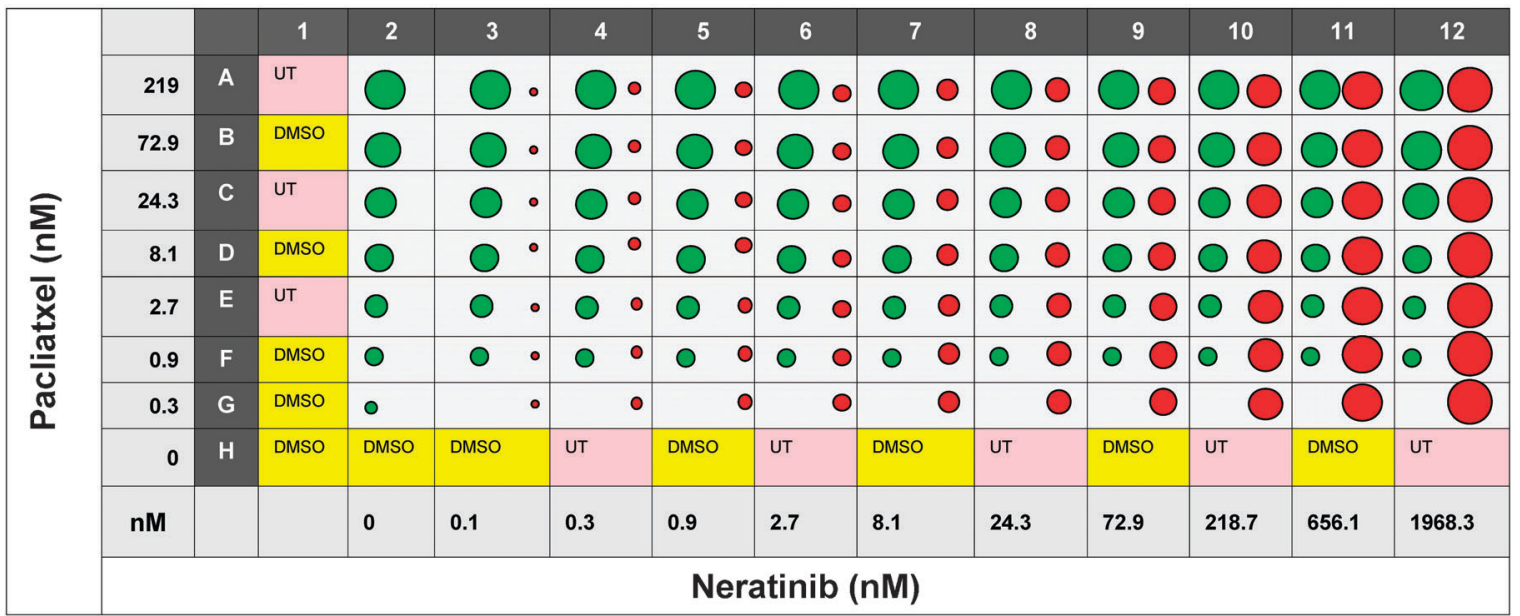

Fig. 4 Testing the combination effects of compounds identified in the screen with neratinib. (A) During network building with "hits" and small molecules, we found that genes increased by paclitaxel are those whose knock-down will cause resistance to neratinib. There are also 4 additional genes previously reported in paclitaxel sensitizer RNAi screen. ${ }^{41}$ (B) Measuring synergy for chemical combinations. Continuous perturbations with sigmoidal response curves can cooperate either to boost the high-dose effect to new levels or to shift the effective concentrations to lower doses, and the optimal dosing ratio is usually not known. ${ }^{51}$ (C) A factorial dose matrix was used to sample all mixtures of two serially diluted single active compounds. 10000 cells per well of a 96 well plate were used to test each compound individually at fixed multiples $(0.3-218 \mathrm{nM})$ for each compound (paclitaxel, cytarabine, vinblastine, and colchicine) and $0.1-1968 \mathrm{nM}$ for neratinib where $\mathrm{IC}_{50}$ for each compound was centered in each drug's concentration window. Controls used for the drug treatments are: DMSO, cells treated with DMSO only and UT, untreated cells, cells were not subjected to either the DMSO or the drugs. Adapted from ref. 60. 
topoisomerase inhibitor etoposide. Thus different drugs impose synthetic effects from those "distant" pathways observed earlier. Overall the taxanes and cytarabine have the strongest synthetic lethal signatures and indication for a combinatorial therapy with neratinib.

In addition to listing paclitaxel as the top scored compound, cMAP also listed other compounds, such as valinomycin that is known to modulate doxorubicin resistance, naringenin, a predominant flavonoid in grapefruit that is known to inhibit cytochrome P450 isoform CYP1A2 and alter the pharmacokinetics of some drugs, and anisomycin, which is known to sensitize metastatic epithelial cells to anoikis and reduce circulating tumor cell implantation in vitro. ${ }^{50}$

\section{Chemical combinations of paclitaxel and cytarabine with neratinib potentiate drug response}

Our RNAi synthetic lethal screen in combination with sub-effective concentrations of neratinib has identified novel targets for drug-combination strategies. In this screen the

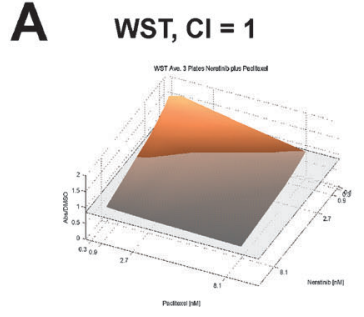

B WST, $\mathrm{Cl}=\mathbf{0 . 7 1}$

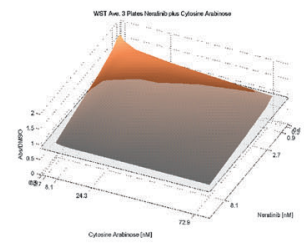

C wST, $\mathrm{Cl}=0.89$

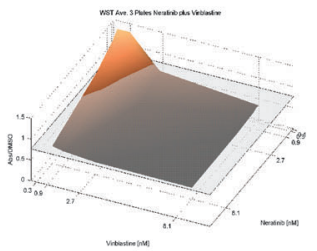

D WST, $\mathrm{Cl}=1$

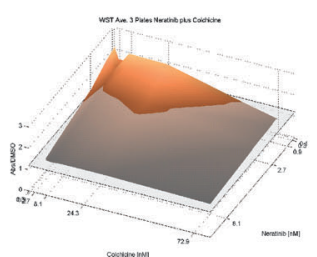

Paclitaxel + Neratinib

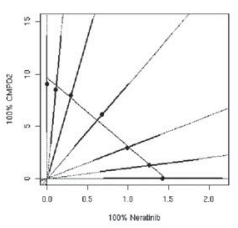

Cytarabine + Neratinib
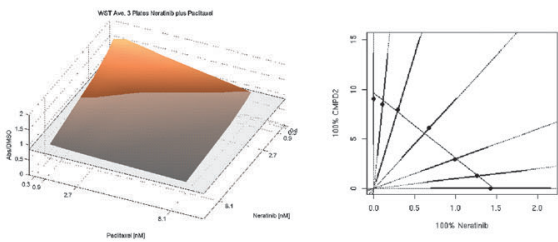

$\mathrm{CTG}, \mathrm{Cl}=0.87$

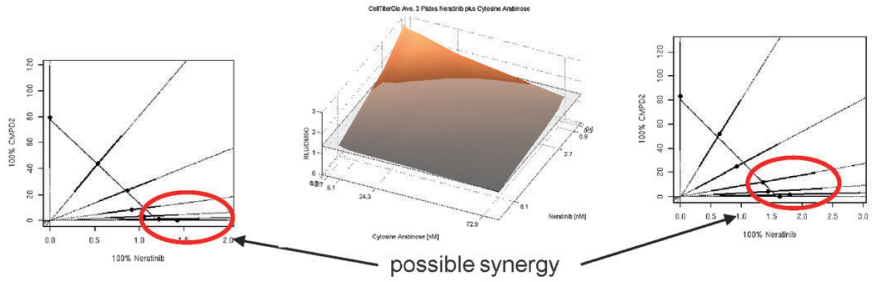

Vinblastine + Neratinib

CTG, $\mathrm{Cl}=1$
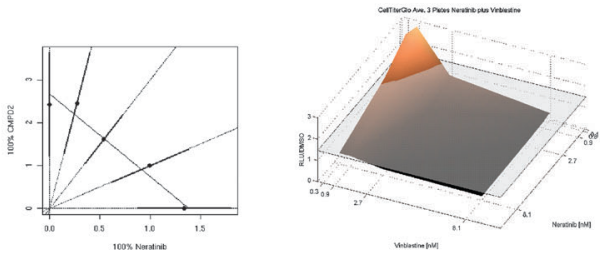

Colchicine + Neratinib
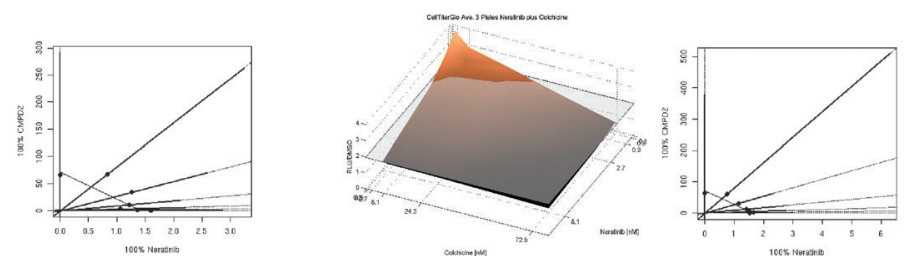

Fig. 5 Determining drug interaction using a dose matrix design with the compounds identified from the synthetic lethal interaction screen. To assess the type of drug:drug interaction, each measurement was compared to expected values derived from the single-compound data along the left and bottom edges of the matrix. The resulting interaction was analyzed using the full-three dimensional response surface or using an isobologram to measure linear dose shifting at a chosen effect level via a combination index (CI). ${ }^{60}$ The orange color represents signal that is above $0.5 \times$ DMSO. The portion of the surface below $0.5 \times$ DMSO is in grey. Isobologram uses the same data. Each isobologram shows the result for a neratinib + second active compound (CMPD2) dose matrix using either cell proliferation (WST) or cell viability (CTG) assays as the endpoint assay readouts averaged over the six replicate plates. Each positive-slope ray corresponds to data from wells with the same \% neratinib composition. The vertical ray corresponds to the CMPD2 dose curve, while the horizontal one is the neratinib only dose curve. The dot is the $\mathrm{IC}_{50}$ value derived from those wells, with the $95 \%$ confidence interval shown in bold. If there is synergy between the compounds, one would expect the dots to lie in an L-shaped or concave curve. The straight line is a best fit to an additivity model although some data showed synergy. Paclitaxel and neratinib showed a strong additive but slight synergy (A) while cytarabine and neratinib showed strong synergistic effect (B). Vinblastine and neratinib (C) and colchicine and neratinib (D) combination were shown to be either additive or synergistic depending on whether cell viability or proliferation assays were used as the end point read outs. CMPD2 = paclitaxel, vinblastine or colchicine. CI: Combination Index (CI). $\mathrm{CI} \approx 1$ : additivity. $\mathrm{CI}<1$ : synergy. 
greatest potentiation was achieved by RNAi-mediated inhibition of microtubulin as well as DNA polymerase associated genes. This has provided a rationale to combine the microtubule inhibitor paclitaxel and the DNA polymerase inhibitor cytarabine with neratinib. To test the synthetic lethal effect induced by these compounds, we used a factorial dose matrix design (Fig. 4) to capture the combination effects of compounds with neratinib on cell growth and viability. In this design, continuous perturbations with sigmoidal response curves can cooperate either to boost the high-dose effect to new levels or to shift the effective concentrations to lower doses. $^{51}$ The resulting interaction can then be analyzed using the full-three dimensional response surface or using an isobologram to measure linear dose shifting at a chosen effect level via a combination index (CI). CI $<1$, CI $=1$, and CI $>1$ represent synergism, additivity, and antagonism of the two agents, respectively. ${ }^{47}$

We functionally-validated the combination of neratinib, microtubulin and DNA polymerase inhibitors as a potential combination treatment regimen in SKBR-3 cells (Fig. 4). Results showed that treatment of SKBR-3 cells with either of the microtubulin inhibitor (paclitaxel) or DNA polymerase inhibitor (cytarabine) led to sensitization of the breast cancer cells to neratinib (Fig. 5) suggesting a strong additive effect with paclitaxel and neratinib $(\mathrm{CI}=1$ for cell proliferation assay and $\mathrm{CI}=0.95$ for cell viability assay) and synergistic effect with cytarabine $(\mathrm{CI}=0.71$ for cell proliferation assay and $\mathrm{CI}=0.87$ for cell viability assay) (Fig. 5).

In conjunction with paclitaxel which blocks microtubule disassembly, we also combined two additional compounds that block microtubule assembly, vinblastine and colchicine, with neratinib. We rationalized that these tubulin inhibitors could show a similar effect as paclitaxel. Our results showed a strong additive effect of vinblastine and neratinib (CI $=0.89$ for cell proliferation assay and $\mathrm{CI}=1$ cell for viability assay), and the colchicine and neratinib combination was shown to be either additive or synergistic depending on assay read outs (Fig. 5, CI $=1$ for cell proliferation assay and CI $=0.71$ for cell viability assay).

\section{Discussion}

In the current study, we interrogated the genetic basis of cancer cell chemo-responsiveness using a genome-wide RNAi library and in the presence of a subeffective concentration of a cancer drug in breast cancer cells. An ErbB2 positive human breast cancer line SKBR-3 was used in the synthetic interaction screen to identify gene targets that specifically reduce cell viability in the presence of subeffective concentrations of neratinib, an irreversible ErbB2 receptor tyrosine kinase inhibitor that is in phase II clinical trial with advanced ErbB2-positive breast cancer and advanced non-small cell lung cancer. ${ }^{19,20,52}$

By using pool-based lentiviral shRNA library approach, we identified candidate RNAi/neratinib-dependent genes whose depletion constitutes synthetic lethality or survival advantage of breast cancer cells. We used a stringent statistical approach using FDR below $0.01 \%$ and identified a panel of genes that represent major focal points of the autonomous response of cancer cells to subeffective concentrations of neratinib (Tables 1 and 2). Our data show that several of these targets sensitize or resist SKBR-3 cells to neratinib at concentrations $\sim 5-8$-fold lower $\left(\mathrm{IC}_{30} 0.39 \mathrm{nM} \text { versus } \mathrm{IC}_{50} 2-3 \mathrm{nM}\right)^{3,16}$ than otherwise required for a significant response, and we identified mechanistic relationships between cancer-associated aberrant gene expression and the basic cellular machinery required for robust mitotic progression.

The vast majority of the genes identified in the screen are implicated in various cellular functions (Tables 1 and 2). Our study suggests that a broad genetic network spanning multiple cellular functions is required to support the cellular proliferation and viability. Many genes in this network could be exploited as potential therapeutic targets as well as targets for combinatorial therapy, as demonstrated by the synthetic lethal effect of their depletion by RNAi.

It is worth mentioning that we could only probe $2 / 3$ of the starting shRNA library since barcode microarrays contain only $\sim 52000$ annotated probes for the library that contains $\sim 74000$ shRNAs. This has resulted in many potential candidate genes not being able to be detected. In addition, the stable selection of cell lines over a two-week period may have selected out some of the targets, and that they would be missing from the neratinib sensitizing gene list.

Our "synthetic lethality" screen identified several "chemosensitizer" genes (Table 1). Our result suggests that breast cancer cells have unique growth and survival requirements where knockdown of these genes via RNAi in combination with neratinib, but not by either alone, leads to chemosensitization of these cells. Targeting such key vulnerabilities is an attractive approach for cancer-selective therapeutics. Our study also demonstrates that highly parallel dropout synthetic lethal screens that use RNAi library covering entire annotated human genome can identify a large number of chemosensitizer or lethal genes representing potential new drug targets. The functional genetic approach demonstrated here presents a rapid, alternative and complementary effort to sequencingbased approaches such as the Cancer Genome Atlas and similar efforts, which focus on physical alterations of the cancer genome.

Complementary to the physical mapping of cancer genomes, functional genomics approaches using genome-scale RNAi screens can identify genetic dependencies of cancer cells. In fact, among the many genes identified and verified in this screen as potential drug targets, only a number of them are known oncogene or tumor suppressor genes, suggesting that there is a much larger set of non-oncogene or tumor suppressor genes that can serve as drug targets in treating cancer. The concept of "nononcogene addiction" (NOA) to describe the extensive dependency of cancer cells on the function of diverse networks of genes which are neither mutated in cancer nor oncogenic for their growth and survival has been previously proposed. ${ }^{53,54}$ This emerging field can potentially reveal new insights on the mechanisms of tumorigenesis, and presents new opportunities for cancer therapeutics that cannot be discovered by examination of genetic alterations in tumors.

RNAi chemosensitization screens reveal information on the possible targets that could improve the selectivity of the drugs 
for cancer cells over normal cells, and as a consequence may lead to an improved therapeutic window. Moreover, these screens have the capability to identify protein targets for drug-combination strategies and identify novel mechanisms of drug sensitivity and resistance. Several recent reports have shown the utility of RNAi chemosensitization screens approach using standard chemotherapy drugs. ${ }^{39,55-58}$ Therefore an expected outcome from our RNAi chemosensitizer screens is the identification of gene products that are targets of currently available compounds that could be used for novel combinatorial therapeutic regimens. Towards this goal, we identified 781 genes that can either increase the sensitivity ("sensitizers") or resistance ("resisters") to subeffective concentrations of neratinib at a relaxed fold change cut-off of 1.5. Pathway analysis identified the significant involvement of the canonical EGFR pathway members, as well as pathways that are very distant from EGFR but converged at the same down-stream effectors, indicating the redundancies in the cellular survival system. The combined local and distal synthetic lethal genes provided us more opportunities for identifying suitable drug combination regimens with neratinib.

Monotherapies are often less effective and usually require the maximum-tolerated dose. For example, a phase I study of neratinib in patients with solid tumors demonstrated that once-daily oral administration was sufficient on the basis of pharmacokinetic consideration. ${ }^{19}$ Among 25 patients who had evaluable tumor responses treated in phase I trial, eight patients $(32 \%)$ had partial responses who had previous treatment with trastuzumab, anthracyclines, and taxanes. ${ }^{19}$ The maximal tolerated dose was $320 \mathrm{mg}$ once daily with the major dose limiting toxicities being diarrhea, fatigue and vomiting. ${ }^{19}$ Because of this, we were interested in identifying pharmaceutical compounds that may be used together with a lower dose of neratinib for a clinically easily acceptable combinatorial therapy regimen. We investigated the roles of small molecules in regulating these genes by text-mining and pathway building studies. We identified Docetaxel/ Paclitaxel as the top candidate, followed by doxycycline and doxorubicin, then COX-1/COX-2 inhibitors, and less significantly the topoisomerase inhibitor etoposide. Paclitaxel stabilizes microtubules and as a result interferes with the normal breakdown of microtubules during cell division. Together with docetaxel, it forms the drug category of the taxanes. Thus different drugs could impose synthetic effects with neratinib from those "distant" pathways observed earlier. Overall the taxanes and cytarabine have the strongest signatures and indication for a combinatorial therapy with neratinib.

As a result of network building with gene "hits" and small molecules, we found that genes increased by a previous paclitaxel siRNA screen ${ }^{41}$ were also enriched (conferred resistance to neratinib) in our screen. We have identified an overlapping set of genes that mediated lethality from this screen and the previously reported siRNA-paclitaxel synthetic lethality screen. ${ }^{41}$ Subsequently, the gene set was searched against findings in the Connectivity Map. This identified the neratinib gene set as "red on red" within the mapping (or synergistic effects predicted by the Map). There were also four additional genes previously reported in the paclitaxel sensitizer RNAi screen ${ }^{41}$ that emerged in our screen (Fig. 4). From these analyses, we identified paclitaxel as an optimal combination agent and confirmed it by in vitro drug combination experiments (Fig. 5). The combination of neratinib and paclitaxel as well as cytarabine showed additive or synergistic inhibitory effects on cell proliferation and viability, respectively (Fig. 5). This is noteworthy because clinical data implicated that neratinib and paclitaxel would have clinical activity in combination. Correlation between ER and PR positive tumors and paclitaxel sensitivity has been demonstrated. ${ }^{59}$ Higher levels of PR and PR mRNA expression correlated with decreased paclitaxel. ${ }^{59}$ Cells from carcinomas with T-stages 3 and 4 were also less sensitive to paclitaxel compared to stages 1 and 2 .

Interestingly, neratinib and paclitaxel have been shown to be active in patients with ErbB2 + metastatic breast cancer (phase II clinical study 203, Wyeth). In this study, a combination dose of neratinib with paclitaxel was shown to be tolerable for patients with solid tumors, and safety and preliminary efficacy were assessed in patients with ErbB2 positive metastatic breast cancer. This combination of $240 \mathrm{mg}$ neratinib and $80 \mathrm{mg} \mathrm{m}^{-2}$ paclitaxel was tolerable with a toxicity profile similar to that observed for neratinib and for paclitaxel as single agents, and had promising antitumor activity in patients with ErbB2 positive breast cancer. ${ }^{21}$ This observation highlights the strong predictive power of genome-wide synthetic-lethal screens to identify productive drug-drug interactions.

Although drugs that target two or more family members (dual- or pan-kinase inhibitors) may be more effective than single-target ones, perturbing the function of multiple ErbB receptors may lead to undesirable toxic effects since each receptor also has its own function in normal tissue. This provides a strong rationale for combining specific ErbB inhibitors with agents that also target the downstream or some other signal transduction cascade such as alternate EGFR/ Neurophilin/VEGFR, insulin receptor (direct and indirect), apoptosis, hypoxia, protein ubiquitination, or cAMP pathways.

For example, recent studies with $\mathrm{PI} 3 \mathrm{~K} / \mathrm{Akt}$ pathway inhibitors have shown synergistic interactions in vitro and in vivo. ${ }^{3}$ The identification of other pathways that modulate sensitivity to these agents will provide additional clues to the mechanism underlying non-responsiveness to ErbB2 inhibitors and provide an opportunity to design clinical trials with rational combinations of drugs to overcome this resistance. Finally, these downstream pathways are valuable to predict responsiveness to the drugs and to provide potential markers to guide the clinical development of novel cancer therapeutics.

Genes emerged from our screen suggest that various pathways interact with the ErbB signaling cascade, including both the insulin-like growth factor, as well as the estrogen receptor signaling cascades (Fig. 6). Several of these cooperating cascades converge at the PI3K signaling node, and because of this central role of the PI3K node and preclinical activity of neratinib against this complex, we hypothesize that the activity of this node may predict those patients who will have meaningful response to neratinib in the clinic. In preclinical 


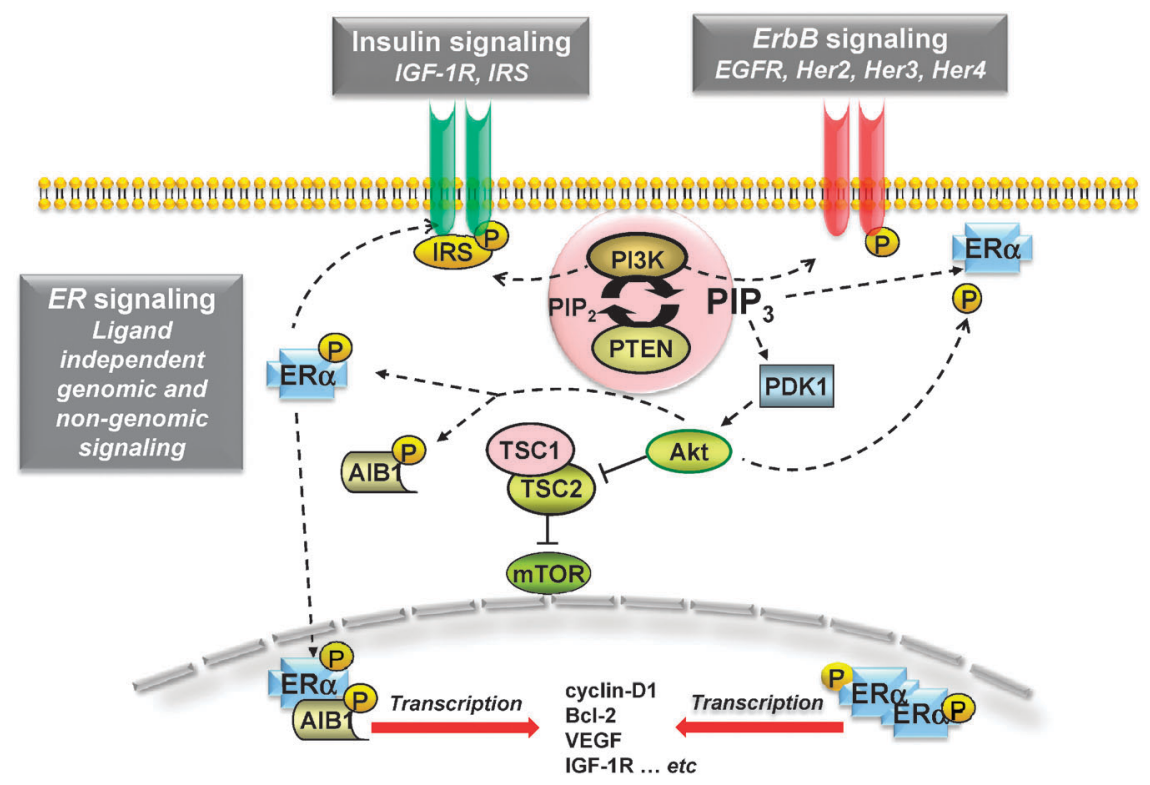

Fig. 6 A hypothetical model for drug-associated biomarker for neratinib. The screening data suggest that various pathways have crosstalk with the ErbB signaling cascade, including both the insulin-like growth factor, as well as the estrogen receptor signaling cascades. Several of these cooperating cascades converge at the PI3K signaling node, and because of this central role of the PI3K node and preclinical activity of neratinib against this complex, we hypothesize that the activity of this node may predict those patients who will have meaningful response to neratinib in the clinic.

models, neratinib was shown to inhibit ErbB receptor complex downstream signaling via the PI3K and MAPK pathways. ${ }^{21}$ It has been proposed that activation of the PI3K signaling pathway may mediate resistance to herceptin or endocrine therapy in breast cancer due to the crosstalks among estrogen receptor, ErbB, and PI3K pathways. ${ }^{21}$ It has been recognized that the estrogen receptor is capable of up-regulating signaling from various cell surface receptors (including the ErbB family); hence, it is logical to investigate the presence or absence of the hormone receptors as well as all of the members of the ErbB cascade. Neratinib has exhibited antitumor efficacy in phase I and II clinical studies in patients with ErbB2 positive breast cancer, including those with or without prior herceptin exposure. ${ }^{19,20}$ Because of this, the upcoming phase III trials is expected to focus on the PI3K signaling node as a potential biomarker to identify the patient population that is predicted to have the best response to neratinib (either following adjuvant herceptin, or in combination with paclitaxel).

The study by Bernards et ll $^{43}$ is an additional support in this direction. In that study, using an unbiased RNAi genetic screen and herceptin, these investigators identified the PTEN gene as a mediator of herceptin resistance in the ErbB2overexpressing breast cancer cell line BT474. Reduced or absent expression of PTEN, a known tumor suppressor, is seen in a significant fraction of human breast malignancies. Because PTEN is a negative regulator of the PI3K pathway (Fig. 6), one might suspect that activation of the PI3K pathway via other means could also lead to a similar herceptin-resistant phenotype.

Collectively, our data demonstrate that RNAi screening is a powerful technology for genome-wide screening of novel interacting targets that can enhance the activity of existing chemotherapeutic agents and should facilitate the identification of effective combination therapies.

\section{Conclusion}

In this study, we carried out a genome-wide RNAi and neratinibdependent "synthetic modulator screen" to identify novel components of ErbB2 and other signaling pathways that specifically reduce cell proliferation or viability in the presence of subeffective concentrations of neratinib in an ErbB2-overexpressing human breast cancer cell line SKBR-3. The screen identified a large number of genes that positively or negatively regulate cell survival and a diverse set of pathways including EGFR, hypoxia, cAMP, and protein ubiquitination and signaling.

Most striking was the identification of novel targets and clinically relevant compounds for combination treatment of human breast cancer cells. Notably, the strong additive effect achieved by neratinib in combination with paclitaxel supports the data obtained in a phase II clinical trial and further supports a phase III trial with this combination in breast cancer patients.

In conclusion, our findings validate the utility of whole genome RNAi synthetic modifier screens for identifying novel drug sensitizing or resistant genes in human breast cancer cells leading to the identification of novel mediators of cellular response to a cancer drug and the development of potential drug combination treatments.

\section{Acknowledgements}

We thank Christina Coughlin for her feedback on the project and Anna Berkenblit, Karl Nocka, Paresh Thakker, and Lih-Ling Lin for critical reading of the article. This study was sponsored by Pfizer Inc. 


\section{References}

1 D. J. Slamon, W. Godolphin, L. A. Jones, J. A. Holt, S. G. Wong, D. E. Keith, W. J. Levin, S. G. Stuart, J. Udove and A. Ullrich, et al., Science, 1989, 244, 707-712.

2 N. E. Hynes and D. F. Stern, Biochim. Biophys. Acta, 1994, 1198, 165-184.

3 S. K. Rabindran, Cancer Lett. (Shannon, Irel.), 2005, 227, 9-23.

4 L. M. Murrow, S. V. Garimella, T. L. Jones, N. J. Caplen and S. Lipkowitz, Breast Cancer Res. Treat., 2010, 122, 347-357.

5 J. D. Brenton, L. A. Carey, A. A. Ahmed and C. Caldas, J. Clin. Oncol., 2005, 23, 7350-7360.

6 W. J. Irvin, Jr. and L. A. Carey, Eur. J. Cancer, 2008, 44, 2799-2805.

7 T. Sorlie, C. M. Perou, R. Tibshirani, T. Aas, S. Geisler, H. Johnsen, T. Hastie, M. B. Eisen, M. van de Rijn, S. S. Jeffrey, T. Thorsen, H. Quist, J. C. Matese, P. O. Brown, D. Botstein, P. Eystein Lonning and A. L. Borresen-Dale, Proc. Natl. Acad. Sci. U. S. A., 2001, 98, 10869-10874.

8 F. Bertucci, P. Finetti, N. Cervera, B. Esterni, F. Hermitte, P. Viens and D. Birnbaum, Int. J. Cancer, 2008, 123, 236-240.

9 E. Charafe-Jauffret, C. Ginestier, F. Monville, P. Finetti, J. Adelaide, N. Cervera, S. Fekairi, L. Xerri, J. Jacquemier, D. Birnbaum and F. Bertucci, Oncogene, 2006, 25, 2273-2284.

10 R. M. Neve, K. Chin, J. Fridlyand, J. Yeh, F. L. Baehner, T. Fevr, L. Clark, N. Bayani, J. P. Coppe, F. Tong, T. Speed, P. T. Spellman, S. DeVries, A. Lapuk, N. J. Wang, W. L. Kuo, J. L. Stilwell, D. Pinkel, D. G. Albertson, F. M. Waldman, F. McCormick, R. B. Dickson, M. D. Johnson, M. Lippman, S. Ethier, A. Gazdar and J. W. Gray, Cancer Cell, 2006, 10, 515-527.

11 T. T. Junttila, R. W. Akita, K. Parsons, C. Fields, G. D. Lewis Phillips, L. S. Friedman, D. Sampath and M. X. Sliwkowski, Cancer Cell, 2009, 15, 429-440.

12 M. A. Cobleigh, C. L. Vogel, D. Tripathy, N. J. Robert, S. Scholl, L. Fehrenbacher, J. M. Wolter, V. Paton, S. Shak, G. Lieberman and D. J. Slamon, J. Clin. Oncol., 1999, 17, 2639-2648.

13 C. L. Vogel, M. A. Cobleigh, D. Tripathy, J. C. Gutheil, L. N. Harris, L. Fehrenbacher, D. J. Slamon, M. Murphy, W. F. Novotny, M. Burchmore, S. Shak, S. J. Stewart and M. Press, J. Clin. Oncol., 2002, 20, 719-726.

14 I. Smith, M. Procter, R. D. Gelber, S. Guillaume, A. Feyereislova, M. Dowsett, A. Goldhirsch, M. Untch, G. Mariani, J. Baselga, M. Kaufmann, D. Cameron, R. Bell, J. Bergh, R. Coleman, A. Wardley, N. Harbeck, R. I. Lopez, P. Mallmann, K. Gelmon, N. Wilcken, E. Wist, P. Sanchez Rovira and M. J. Piccart-Gebhart, Lancet, 2007, 369, 29-36.

15 T. Fujita, H. Doihara, K. Kawasaki, D. Takabatake, H. Takahashi, K. Washio, K. Tsukuda, Y. Ogasawara and N. Shimizu, Br. J. Cancer, 2006, 94, 247-252.

16 S. K. Rabindran, C. M. Discafani, E. C. Rosfjord, M. Baxter, M. B. Floyd, J. Golas, W. A. Hallett, B. D. Johnson, R. Nilakantan, E. Overbeek, M. F. Reich, R. Shen, X. Shi, H. R. Tsou, Y. F. Wang and A. Wissner, Cancer Res., 2004, 64, 3958-3965.

$17 \mathrm{H}$. R. Tsou, E. G. Overbeek-Klumpers, W. A. Hallett, M. F. Reich, M. B. Floyd, B. D. Johnson, R. S. Michalak, R. Nilakantan, C. Discafani, J. Golas, S. K. Rabindran, R. Shen, X. Shi, Y. F. Wang, J. Upeslacis and A. Wissner, J. Med. Chem., 2005, 48, 1107-1131.

18 Y. Minami, T. Shimamura, K. Shah, T. LaFramboise, K. A. Glatt, E. Liniker, C. L. Borgman, H. J. Haringsma, W. Feng, B. A. Weir, A. M. Lowell, J. C. Lee, J. Wolf, G. I. Shapiro, K. K. Wong, M. Meyerson and R. K. Thomas, Oncogene, 2007, 26, 5023-5027.

19 K. K. Wong, P. M. Fracasso, R. M. Bukowski, T. J. Lynch, P. N. Munster, G. I. Shapiro, P. A. Janne, J. P. Eder, M. J. Naughton, M. J. Ellis, S. F. Jones, T. Mekhail, C. Zacharchuk, J. Vermette, R. Abbas, S. Quinn, C. Powell and H. A. Burris, Clin. Cancer Res., 2009, 15, 2552-2558.

20 H. J. Burstein, Y. Sun, L. Y. Dirix, Z. Jiang, R. Paridaens, A. R. Tan, A. Awada, A. Ranade, S. Jiao, G. Schwartz, R. Abbas, C. Powell, K. Turnbull, J. Vermette, C. Zacharchuk and R. Badwe, J. Clin. Oncol., 2010, 28, 1301-1307.

21 L. Chow, S. Gupta, D. L. Hershman, Jiang, R. Epstein, Coughlin, A. Freyman, Y. Zhao, R. Abbas and A. Awada, J. Clin. Oncol., 2009, 27, 3557, 2009 ASCO Annual Meeting Proceedings.
22 D. M. Dykxhoorn, C. D. Novina and P. A. Sharp, Nat. Rev. Mol. Cell Biol., 2003, 4, 457-467.

23 G. Meister and T. Tuschl, Nature, 2004, 431, 343-349.

24 P. D. Zamore and B. Haley, Science, 2005, 309, 1519-1524.

25 A. A. Seyhan, Eur. Biopharm. Rev., 2010, 13-24.

26 A. A. Seyhan and T. E. Ryan, Curr. Pharm. Biotechnol., 2010, 11, 735-756.

27 A. Bender and J. R. Pringle, Mol. Cell. Biol., 1991, 11 $1295-1305$.

28 A. Simons, N. Dafni, I. Dotan, Y. Oron and D. Canaani, Genome Res., 2001, 11, 266-273.

29 B. R. Stockwell, S. J. Haggarty and S. L. Schreiber, Chem. Biol., 1999, 6, 71-83.

30 A. A. Seyhan and T. E. Ryan, Curr. Pharm. Biotechnol., 2010, 11, 735-756.

31 R. Bernards, T. R. Brummelkamp and R. L. Beijersbergen, Nat. Methods, 2006, 3, 701-706.

32 J. Downward, Oncogene, 2004, 23, 8376-8383.

33 T. F. Westbrook, F. Stegmeier and S. J. Elledge, Cold Spring Harb. Symp. Quantum Biol., 2005, 70, 435-444.

34 M. R. Schlabach, J. Luo, N. L. Solimini, G. Hu, Q. Xu, M. Z. Li, Z. Zhao, A. Smogorzewska, M. E. Sowa, X. L. Ang, T. F. Westbrook, A. C. Liang, K. Chang, J. A. Hackett, J. W. Harper, G. J. Hannon and S. J. Elledge, Science, 2008, $319,620-624$

35 T. R. Brummelkamp, A. W. Fabius, J. Mullenders, M. Madiredjo, A. Velds, R. M. Kerkhoven, R. Bernards and R. L. Beijersbergen, Nat. Chem. Biol., 2006, 2, 202-206.

36 J. M. Silva, K. Marran, J. S. Parker, J. Silva, M. Golding, M. R. Schlabach, S. J. Elledge, G. J. Hannon and K. Chang, Science, 2008, 319, 617-620.

37 C. Scholl, S. Frohling, I. F. Dunn, A. C. Schinzel, D. A. Barbie, S. Y. Kim, S. J. Silver, P. Tamayo, R. C. Wadlow, S. Ramaswamy, K. Dohner, L. Bullinger, P. Sandy, J. S. Boehm, D. E. Root, T. Jacks, W. C. Hahn and D. G. Gilliland, Cell (Cambridge, Mass.), 2009, 137, 821-834.

38 V. N. Ngo, R. E. Davis, L. Lamy, X. Yu, H. Zhao, G. Lenz, L. T. Lam, S. Dave, L. Yang, J. Powell and L. M. Staudt, Nature, 2006, 441, 106-110.

39 N. C. Turner, C. J. Lord, E. Iorns, R. Brough, S. Swift, R. Elliott, S. Rayter, A. N. Tutt and A. Ashworth, EMBO J., 2008, 27, $1368-1377$.

40 D. O. Azorsa, I. M. Gonzales, G. D. Basu, A. Choudhary, S. Arora, K. M. Bisanz, J. A. Kiefer, M. C. Henderson, J. M. Trent, D. D. Von Hoff and S. Mousses, J. Transl. Med., 2009, 7, 43.

41 A. W. Whitehurst, B. O. Bodemann, J. Cardenas, D. Ferguson, L. Girard, M. Peyton, J. D. Minna, C. Michnoff, W. Hao, M. G. Roth, X. J. Xie and M. A. White, Nature, 2007, 446, 815-819.

42 J. Luo and S. J. Elledge, Nature, 2008, 453, 995-996.

43 K. Berns, H. M. Horlings, B. T. Hennessy, M. Madiredjo, E. M. Hijmans, K. Beelen, S. C. Linn, A. M. Gonzalez-Angulo, K. Stemke-Hale, M. Hauptmann, R. L. Beijersbergen, G. B. Mills, M. J. van de Vijver and R. Bernards, Cancer Cell, 2007, 12 , 395-402.

44 J. Lehar, G. R. Zimmermann, A. S. Krueger, R. A. Molnar, J. T. Ledell, A. M. Heilbut, G. F. Short, 3rd, L. C. Giusti, G. P. Nolan, O. A. Magid, M. S. Lee, A. A. Borisy, B. R. Stockwell and C. T. Keith, Mol. Syst. Biol., 2007, 3, 80 .

45 T. C. Chou, Pharmacol. Rev., 2006, 58, 621-681.

46 E. Iorns, C. J. Lord, A. Grigoriadis, S. McDonald, K. Fenwick, A. Mackay, C. A. Mein, R. Natrajan, K. Savage, N. Tamber, J. S. Reis-Filho, N. C. Turner and A. Ashworth, PLoS One, 2009, 4, e5120.

47 P. Bali, M. Pranpat, R. Swaby, W. Fiskus, H. Yamaguchi, M. Balasis, K. Rocha, H. G. Wang, V. Richon and K. Bhalla, Clin. Cancer Res., 2005, 11, 6382-6389.

48 G. K. Smyth and T. Speed, Methods, 2003, 31, 265-273.

49 J. Lamb, E. D. Crawford, D. Peck, J. W. Modell, I. C. Blat, M. J. Wrobel, J. Lerner, J. P. Brunet, A. Subramanian, K. N. Ross, M. Reich, H. Hieronymus, G. Wei, S. A. Armstrong, S. J. Haggarty, P. A. Clemons, R. Wei, S. A. Carr, E. S. Lander and T. R. Golub, Science, 2006, 313, 1929-1935. 
50 I. A. Mawji, C. D. Simpson, M. Gronda, M. A. Williams, R. Hurren, C. J. Henderson, A. Datti, J. L. Wrana and A. D. Schimmer, Cancer Res., 2007, 67, 8307-8315.

51 J. Lehar, B. R. Stockwell, G. Giaever and C. Nislow, Nat. Chem. Biol., 2008, 4, 674-681.

52 L. V. Sequist, B. Besse, T. J. Lynch, V. A. Miller, K. K. Wong, B. Gitlitz, K. Eaton, C. Zacharchuk, A. Freyman, C. Powell, R. Ananthakrishnan, S. Quinn and J. C. Soria, J. Clin. Oncol., 2010, 28, 3076-3083.

53 J. Luo, N. L. Solimini and S. J. Elledge, Cell (Cambridge, Mass.), 2009, 136, 823-837.

54 N. L. Solimini, J. Luo and S. J. Elledge, Cell (Cambridge, Mass.), 2007, 130, 986-988.

55 V. Giroux, J. Iovanna and J. C. Dagorn, FASEB J., 2006, 20, 1982-1991.
56 E. Iorns, C. J. Lord, N. Turner and A. Ashworth, Nat. Rev. Drug Discovery, 2007, 6, 556-568.

57 J. P. MacKeigan, L. O. Murphy and J. Blenis, Nat. Cell Biol., 2005, 7, 591-600.

58 C. Swanton, M. Marani, O. Pardo, P. H. Warne, G. Kelly, E. Sahai, F. Elustondo, J. Chang, J. Temple, A. A. Ahmed, J. D. Brenton, J. Downward and B. Nicke, Cancer Cell, 2007, 11, 498-512.

59 M. Schmidt, E. Bremer, D. Hasenclever, A. Victor, M. Gehrmann, E. Steiner, I. B. Schiffer, S. Gebhardt, H. A. Lehr, M. Mahlke, M. Hermes, A. Mustea, B. Tanner, H. Koelbl, H. Pilch and J. G. Hengstler, Br. J. Cancer, 2007, 96, 241-247.

60 J. Lehar, A. S. Krueger, W. Avery, A. M. Heilbut, L. M. Johansen, E. R. Price, R. J. Rickles, G. F. Short, 3rd, J. E. Staunton, X. Jin, M. S. Lee, G. R. Zimmermann and A. A. Borisy, Nat. Biotechnol., 2009, 27, 659-666. 